the concept of zinc deficiency anemia in healthy school children which was not described before. Smaller head circumferences in zinc deficient children might contribute to cognitive deficiencies.

\section{NUTRITIONAL STATUS AND FEEDING PROBLEMS IN PATIENTS WITH CEREBRAL PALSY}

doi:10.1136/archdischild-2012-302724.1506

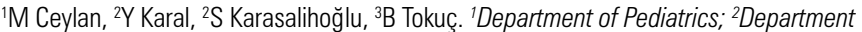
of Pediatrics, Division Pediatric Neurology; ${ }^{3}$ Department of Public Health, Medical Faculty of Trakya University, Edirne, Turkey

Aim The nutritional status and feeding problems in children with cerebral palsy results in growth retardation, increased morbidity and mortality. The aim of this study is to evaluate the nutritional status and feeding problems of cerebral palsy patients followed up by Medical Faculty of Trakya University, Department of Pediatrics, Division Pediatric Neurology.

Methods The medical histories and full physical examination findings of 66 cerebral palsy patients were recorded as weight, height, body mass indeks, circumference measurements of head, arm, chest, fathom length, biacromial length, lower leg length, and subcutaneous fat thickness of triceps and subscapular region. The 3-day diet for each patient was evaluated by a special computer program called BEBIS. Patients' blood hemoglobin, iron levels and binding capacity and serum ferritin, vitamin B12, folic acid levels were also evaluated.

Results The majority of patients were Spastic Cerebral Palsy (\%89), \%6 were Ataxic and \%5 were Mixt type (Quadriplegic/ Distonic). The weight, body mass index, subcutaneous fat thickness of subscapular region, biacromial length, arm-chest circumference, blood iron levels, dietary calory and fiber intakes of patients with severly affected cerebral palsy, expecially in the Quadriplegic/Mixt types, were lower than the mild and moderately affected ones.

Conclusions The nutritional status and dietary intakes of the severly affected cerebral palsy are worse than the others in our study, as mentioned in the literature. To prevent complications a multidisciplinary approach and close follow up have to be done.

\section{EFFECTS OF LAMOTRIGINE ON THE LANGUAGE AND PROBLEM SOLVING ABILITIES IN NEWLY DIAGNOSED PEDIATRIC EPILEPTIC PATIENTS}

doi:10.1136/archdischild-2012-302724.1507

'SJ Kim, ${ }^{2} \mathrm{SH}$ Chae, ${ }^{2} \mathrm{YM}$ Choi, ${ }^{3} \mathrm{KS}$ Kim. 'Department of Pediatrics; ${ }^{2}$ Department of Clinical Speech Pathology, Chonbuk National University Medical School; '3Jeonju Public Health Center, Jeonju, Republic of Korea

Purpose The purpose of this study was to investigate the effects of lamotrigine on language and problem solving abilities in newly diagnosed pediatric epileptic patients.

Methods Sixty-seven newly diagnosed pediatric epileptic patients. (Male:Female $=43: 24$, Mean age $: 8$ y $9 \mathrm{~m} \pm 2 \mathrm{y} 4 \mathrm{~m}$ ), who were investigated from 2006 to July 2011. We performed a standardized full articulation tests and Peabody picture vocabulary test-revised. Test of Problem Solving (TOPS), Mean Length of Utterance in words (MLU-w), comparison of Precise Articulation, Computerized Speech Lab were used to assess the language function before and after initiation of lamotrigine. Starting dosage of lamotrigine was $1 \mathrm{mg} / \mathrm{kg}$ for the first $7-14$ days; increased to $2 \mathrm{mg} / \mathrm{kg}$ for the next 14 days and increased up to $7 \mathrm{mg} / \mathrm{kg} /$ day (or $200 \mathrm{mg} /$ day).

Results First, TOPS showed that the abilities of problem solving were not injured after initiation of lamotrigine (32.9 \pm 13.0 vs $34.5 \pm 12.5, \mathrm{P}>0.05)$. All parameters: Causal reason (11.6 \pm 4.5 vs $12.1 \pm 4.2)$, solution ratiocination $(13.4 \pm 5.4$ vs $13.6 \pm 5.6)$, beginning guess $(7.9 \pm 4.2$ vs $8.7 \pm 4.0)$ were not changed after initiation of lamotrigine. Second, MLU-w did not reduce after taking medicine
(4.2 \pm 1.4 vs $4.2 \pm 1.3)$. Third, the receptive language function was significantly improved after taking lamotrigine in PPVT (8y $4 \mathrm{~m} \pm 2 y$ $4 \mathrm{~m}$ vs $8 \mathrm{y} 10 \mathrm{~m} \pm 2 \mathrm{y} 4 \mathrm{~m}, \mathrm{P}<0.01)$. However, there were no significant changes in percentages of precise articulation and error pattern of consonants after taking lamotrigine ( $98.3 \%$ to $99.1 \%, \mathrm{P}>0.05)$.

Conclusions Our results suggest that lamotrigine can be used without significant negative effects on language function. Moreover, language functions, especially receptive language, were improved after lamotrigine initiation.

\section{SPECTRUM OF NEURAL TUBE DEFECTS AFTER PRENATAL ANTIEPILEPTIC DRUG EXPOSURE: EXTENSIVE CASE SERIES}

doi:10.1136/archdischild-2012-302724.1508

${ }^{1} \mathrm{MH}$ van Dijk, ${ }^{2} \mathrm{~S}$ Bulk, ${ }^{3} \mathrm{ACC}$ van Oppen, ${ }^{1,4} \mathrm{ACG}$ Egberts, ${ }^{1,4}$ ER Heerdink, ${ }^{2,5} \mathrm{D}$ Lindhout. ${ }^{1}$ Department of Clinical Pharmacy; ${ }^{2}$ Department of Medical Genetics; ${ }^{3}$ Department of Obstetrics and Perinatology, University Medical Center Utrecht; ${ }^{4}$ Department of Pharmacoepidemiology and Clinical Pharmacology, Utrecht Institute for Pharmaceutical Sciences; ${ }^{5}$ SEIN - Epilepsy Institute, Utrecht, The Netherlands

Background Most pregnant women with chronic active epilepsy need to use antiepileptic drugs (AEDs) during pregnancy to prevent epileptic seizures that may threaten maternal and fetal well-being. Valproic acid (VPA) and carbamazepine (CBZ) have been associated with an increased risk of neural tube defects (NTDs) in the exposed fetus.

Aim To investigate the spectrum of neural tube defects and associated central nervous system (CNS) and non-CNS malformations after prenatal exposure to CBZ and/or VPA.

Methods NTDs in pregnancies in which CBZ and/or VPA were used during the first trimester were collected from 1970-2012 in the Netherlands. Type and location of the NTDs, associated CNS and non-CNS major malformations and relevant patient characteristics were analysed.

Results 87 pregnancies were included. NTDs after exposure to CBZ or VPA were mostly caudally located, whereas a combination of CBZ and VPA was associated with a location shift of the NTD to the rostral side (Figure 1). There were no differences between CBZ and VPA in the percentage of associated CNS malformations and non-CNS malformations circa $75 \%$ and $45 \%$.

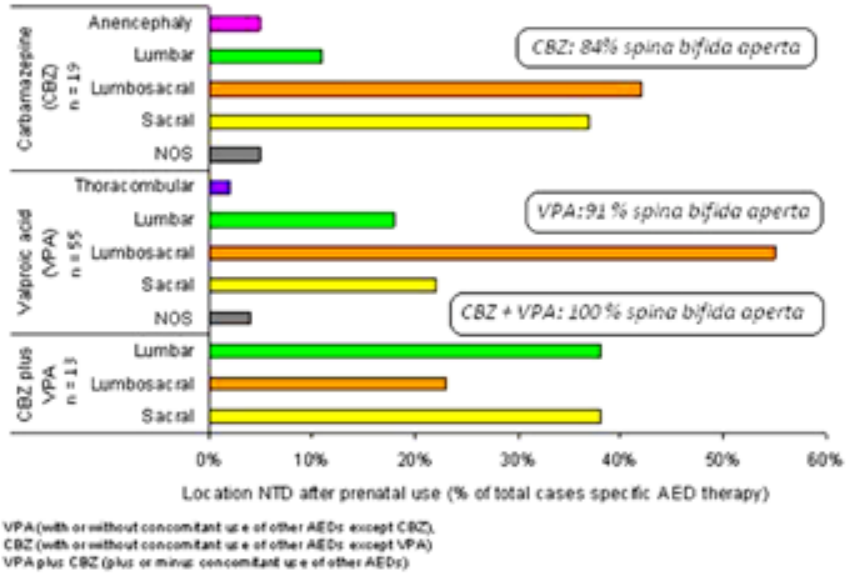

Abstract 1508 Figure 1 Antiepileptic drugs versus type and location of NTDs

Conclusions The combination of VPA and CBZ shows a tendency towards a more rostral location (lumbar) which may have more severe functional consequences. Current findings confirm that NTDs associated fetal exposure to VPA and/or CBZ are serious and frequently accompanied by other CNS and non-CNS malformations. 
Funding Netherlands Epilepsy Foundation (NEF) grant nr 03.18, University Medical Center Utrecht [NL] - institutional Genvlag grant.

\section{PREVALENCE AND CLASSIFICATION OF EPILEPSY AMONG CHILDREN IN A RURAL TEACHING HOSPITAL OF WESTERN INDIA}

doi:10.1136/archdischild-2012-302724.1509

${ }^{1} \mathrm{NH}$ Thacker, 'L Dalal, 'NM Kharod, 1,2SM Nimbalkar. 'Department of Pediatrics, Pramukhswami Medical College; ${ }^{2}$ Central Research Services, Charutar Arogya Mandal, Anand, India

Background and Aims To study Prevalence of Active Epilepsy in children and Clinical pattern of seizures among epileptics. Classification of epilepsy and epileptic syndromes was done according to ICEES,1989.

Methods All 0-18 year old patients; visiting Hospital during the study period. The ones with Active Epilepsy were studied for clinical pattern of seizures and classification of epilepsy.

Results 4981 patients visited the hospital which included 2893 (58\%) males and 2088 (42\%) females. Active Epilepsy was found in 41 boys and 19 girls. Crude Prevalence was 12 per 1000. Gender specific prevalence was higher in males $14.1 / 1000$ as compared to females 9.1/1000. Age specific prevalence was maximum 19.2/1000 in age group of 5-10 yrs. Maximum patients (25\%) had their seizure debut in First year of life. Secondarily generalized seizures were the most common type of seizures with 20 (33.3\%) of patients having it as the main seizure type. Wests syndrome and Lennox Gastaut syndrome were the most common generalized epileptic syndromes. $20 \%$ had Idiopathic Epilepsy, 30\% probably Symptomatic (earlier Cryptogenic) and $50 \%$ had Symptomatic epilepsy. Perinatal asphyxia accounted for $36.7 \%$ of symptomatic epilepsies. $43.3 \%$ of patients had neurohandicaps of which CP alone accounted for $53.8 \%$. $31.7 \%$ of the patients were on multiple AEDs, $30 \%$ were on Valproate alone at the time of presentation.

Conclusions This study emphasizes the fact that perinatal asphyxia, CNS infections are major contributing factors to childhood epilepsy also leading to a higher percentage of Wests syndromes and LG syndrome which is not the case in western studies.

\section{A STUDY OF THE VALUE OF SLEEP EEG RECORD IN PREDICTING SEIZURE RECURRENCE IN CHILDREN WITH A SINGLE AFEBRILE SEIZURE}

doi:10.1136/archdischild-2012-302724.1510

1,2J Chukwu, ${ }^{3 P}$ Gallagher, ${ }^{3} \mathrm{~B}$ McCoy, ${ }^{3} \mathrm{D}$ Webb. 'Molecular \& Cellular Therapeutics, Royal College of Surgeons; ${ }^{2}$ Clinical Research Unit, National Children's Research Centre; ${ }^{3}$ Neurology, Our Lady's Children's Hospital, Dublin, Ireland

Aims The main aim of the study was to determine the value of a sleep EEG recording in predicting the risk of epilepsy in children with a single afebrile seizure.

Methods A retrospective review of 69 children who underwent sleep EEG recordings in 2007 following their first afebrile seizure was undertaken. Parents were sent a questionnaire on seizure recurrence, diagnosis of epilepsy, anti-epileptic medication use, further EEGs and school progress.

Results Fifty two (52) questionnaires (75\%) were completed and returned. Twenty three of the fifty two children (44\%) have had one or more further seizures $-7 / 25(28 \%)$ of those with normal sleep EEG recordings and 14/21 (67\%) of those who had abnormal sleep EEG recordings. 6/52 (12\%) of the respondents had suspicious EEG and 2 of these have had further seizures. 12/21 (57\%) of those with abnormal sleep EEGs compared with 5/25(20\%) of those with normal EEG have had multiple further seizures.
Pearson-Chi Square revealed a significant relationship between abnormal EEG and the development of further seizures $(p<0.01)$. The odds of having further seizures if the EEG was abnormal was 4.9 (95\% C.I 1.5-16.1).

Conclusions Sleep EEG is a useful tool in predicting the risk of developing epilepsy in children with first afebrile seizure.

\section{DOES NEPHROTOXICITY EXIST IN EPILEPTIC PATIENTS ON VALPROATE OR CARBAMAZEPINE THERAPY ? (A PRELIMINARY STUDY)}

doi:10.1136/archdischild-2012-302724.1511

${ }^{1} \mathrm{C}$ Havali, ${ }^{1} \mathrm{~K}$ Gücüyener, ${ }^{2} \mathrm{~N}$ Buyan, 'E Gürkaş, 'E Demir, 'A Serdaroğlu. 'Pediatric Neurology; ${ }^{2}$ Pediatric Nephrology, Gazi University Faculty of Medicine, Ankara, Turkey

Objective The aim of the study was to investigate renal glomerular and tubular side effects of valproate and carbamazepine if exists, in children who are on these antiepileptic drugs between 6 months and two years of time.

Method A prospective study was performed on epileptic children (primary generalized and partial epilepsy) under valproate (n:30), carbamazepine (n:24) treatments and healty control group (n:26). The serum creatinine, Cystatin $C$ levels and urinary excretion of $\mathrm{N}$-acetyl- $\beta$-D-glucosaminidase levels were taken at the beginning of the study and after 6 months of therapy. Three Glomerular filtration rate formulae with creatinine, Cystatin $\mathrm{C}$ and combined of all of were used to determine glomerular filtration functions.

Results Serum creatinine, Cystatin C levels of patients and glomerular filtration rate values were in the normal range according to patient ages and healthy control group. However urinary $\mathrm{N}$-acety$\beta$-D-glucosaminidase/creatinine levels were higher in both groups (valproate 6.1 \pm 5 ) (carbamazepine 3.14 \pm 1.97 ) when compared to the levels of the control group $(2.6 \pm 1.3)(p<0.05)$ Although this is a preliminary study; bearing in mind that patients on both antiepileptic drugs may have a tubulotoxicity risk; renal function test should also be checked in during the treatment of all patients with special emphasis when there is a renal or systemic disease co-existing.

\section{VALIDITY OF THE CLINICAL NEUROLOGICAL STATE IN DIAGNOSING DIABETIC PERIPHERAL NEUROPATHY}

doi:10.1136/archdischild-2012-302724.1512

1,21 Hoeliner, 'V Haslinger, 'J Lütschg, 'G Müller, 'D Seick, ${ }^{3} \mathrm{~J}$ Fussenegger, ${ }^{3} \mathrm{U}$ Zanier, ${ }^{2,4} \mathrm{C}$ Saely, ${ }^{2,4} \mathrm{H}$ Drexel, 'B Simma. 'Paediatrics, Academic Teaching Hospital, Feldkirch, Austria; '2Private University of the Principality of Liechtenstein, Triesen, Liechtenstein; ${ }^{3}$ Krankenhaus Dornbirn, Dornbirn; ${ }^{4}$ Vorarlberg Institute for Vascular Investigation and Treatment, Feldkirch, Austria

Objective We aimed to evaluate the prevalence of diabetic peripheral neuropathy (DPN) in children and adolescents with type 1 diabetes mellitus (T1DM) and examine whether the clinical neurological state validly diagnoses DPN as compared to the gold standard of nerve conduction velocity (NCV) in these patients.

Methods We measured NCV in an unselected consecutive series of patients aged 8-18 years who had been suffering from T1DM for at least one year. The clinical neurological state of these patients was examined using a protocol including ankle reflex, vibration, pinprick, and temperature testing as well as a standardized questionnaire; neuropathy disability scores (NDS) and neuropathy symptom scores (NSS) were assessed.

Results Of our 39 patients, six (15\%) had clinically evident DPN, whereas NCV testing revealed DPN in 15 (38\%) patients. Sensitivity and specificity of the clinical neurological exam for the diagnosis of DPN as compared to the gold standard of pathological NCV were $40 \%$ and $100 \%$, respectively. The corresponding positive and negative predictive values were $100 \%$ and $72.7 \%$, respectively. 